\title{
The Effect of Pitch Accenting on Pronoun Referent Resolution
}

\author{
Janet Cahn \\ Massachusetts Institute of Technology \\ Cambridge, MA 02139 \\ USA \\ cahn@media.mit.edu
}

\begin{abstract}
By strictest interpretation, theories of both centering and intonational meaning fail to predict the existence of pitch accented pronominals. Yet they occur felicitously in spoken discourse. To explain this, I emphasize the dual functions served by pitch accents, as markers of both propositional (semantic/pragmatic) and attentional salience. This distinction underlies my proposals about the attentional consequences of pitch accents when applied to pronominals, in particular, that while most pitch accents may weaken or reinforce a cospecifier's status as the center of attention, a contrastively stressed pronominal may force a shift, even when contraindicated by textual features.
\end{abstract}

\section{Introduction}

To predict and track the center of attention in discourse, theories of centering (Grosz et al., 1983; Brennan et al., 1987; Grosz et al., 1989) and immediate focus (Sidner, 1986) rely on syntactic and grammatical features of the text such as pronominalization and surface sentence position. This may be sufficient for written discourse. For oral discourse, however, we must also consider the way intonation affects the interpretation of a sentence, especially the cases in which it alters the predictions of centering theories. I investigate this via a phenomenon that, by the strictest interpretation of either centering or intonation theories, should not occur - the case of pitch accented pronominals.

Centering theories would be hard pressed to predict pitch accents on pronominals, on grounds of redundancy. To bestow an intonational marker of salience (the pitch accent) on a textual marker of salience (the pronominal) is unnecessarily redundant and especially when textual features correctly predict the focus of attention.

Intonational theories would be similarly hard pressed, but on grounds of information quality and efficient use of limited resources. Given the serial and ephemeral nature of speech and the limits of working memory, it is most expedient to mark as salient the information-rich nonpronominals, rather than their semantically impoverished pronominal stand-ins. To do otherwise is an injudicious use of an attentional cue.

However, when uttered with contrastive stress on the pronouns,

(1) John introduced Bill as a psycholinguist and then $\mathrm{HE}$ insulted HIM.

(after Lakoff, 1971) is felicitously understood to mean that after a slanderous introduction, Bill retaliated in kind against John.

What makes (1) felicitous is that the pitch accents on the pronominals contribute attentional information that cannot be gleaned from text alone. This suggests an attentional component to pitch accents, in addition to the propositional component explicated in Pierrehumbert and Hirschberg (1990). In this paper, I combine their account of pitch accent semantics with Grosz, Joshi and Weinstein's (1989) account of centering to yield insights into the phenomenon of pitch accented pronominals, and the attentional consequences of pitch accents in general. The relevant claims in PH90 and GJW89 are reviewed in the next two sections.

\section{Pitch accent semantics}

A pitch accent is a distinctive intonational contour applied to a word to convey sentential stress (Bolinger, 1958; Pierrehumbert, 1980). PH90 catalogues six pitch accents, all combinations of high (H) and low $(L)$ pitch targets, and structured as a main tone and an optional leading or trailing tone. The form of the accent - $\mathrm{L}, \mathrm{H}, \mathrm{L}+\mathrm{H}$ or $\mathrm{H}+\mathrm{L}$ - informs about the operation that would relate the salient item to the mutual beliefs ${ }^{1}$ of the conversants; the main tone either commits $\left(\mathrm{H}^{*}\right)$ or fails to commit

\footnotetext{
${ }^{1}$ Mutual beliefs: propositions expressed or implied by the discourse, and which all conversants believe each other to accept as true and relevant same (Clark and Marshall, 1981).
} 
$\left(L^{*}\right)$ to the salience of the proposition itself, or the relevance of the operation.

- $\mathrm{H}^{*}$ predicates a proposition as mutually believed, and proclaims its addition to the set of mutual beliefs; $\mathrm{L}^{*}$ fails to predicate a proposition as mutually believed. As PH90 points out, failure to predicate has contradictory sources: the proposition has already been predicated as mutually believed; or, the speaker, but not the hearer, is prevented from predication (perhaps by social constraints); or the speaker actively believes the salient proposition to be false.

- $\mathrm{H}+\mathrm{L}$ evokes an inference path. $\mathrm{H}^{*}+\mathrm{L}$ commits to the existence of inference path that would support the proposition as mutually believed, indicates that it can be found or derived from the set of mutual beliefs; $\mathrm{H}+\mathrm{L}^{*}$ conveys uncertainty about the existence of such a path.

- $\mathrm{L}+\mathrm{H}$ evokes a scale or ordered set to which the accented constituent belongs: $\mathrm{L}+\mathrm{H}^{*}$ commits to the salience of the scale, and is typically used to convey contrastive stress; $\mathrm{L}^{*}+\mathrm{H}$ also evokes a scale but fails to commit to its salience, e.g., conveying uncertainty about the salience of the scale with regard to the accented constituent.

\section{Centering structures and operations}

To explain how speakers move an entity in and out of the center of [mutual] attention, GJW89 formalizes attentional operations with two computational structures - the forward-looking center list (Cf) and the backward-looking center (the $\mathrm{Cb}$ ). Cf is a partially ordered list of centering candidates; ${ }^{2}$ the $\mathrm{Cb}$, at the head of $\mathrm{Cf}$, is the current center of attention.

After each utterance, one of three operations are possible:

- The $\mathrm{Cb}$ retains both its position at the head of $\mathrm{Cf}$ and its status as the $\mathrm{Cb}$; therefore it continues as the center in the next utterance.

- The $\mathrm{Cb}$ retains its centered status for the current utterance but its rank is lowered - it no longer resides at the head of $\mathrm{Cf}$ and therefore ceases to be the center in the next utterance.

- The $\mathrm{Cb}$ loses both its centered status and ranking in the current utterance as attention shifts to a new center.

In addition, GJW89 constrains pronominalization such that no element in an utterance can be realized as a pronoun unless the $\mathrm{Cb}$ is also realized as a pronoun, and imposes a preference ordering for operations on $\mathrm{Cf}$, such that the least reordering is always preferred. That is, a sequence of continuations

\footnotetext{
${ }^{2}$ For simplicity's sake, we assume the items in $\mathrm{Cf}$ to be words and phrases; in actuality, they may be nonlexical representations of concepts, or some hybrid of lexical, conceptual and sensory data.
}

is preferred over a sequence of retentions, which is preferred over a sequence of shifts.

\section{When intonation and centering collide}

My synthesis of the claims in PH90 and GJW89 produces an attentional interpretation of pitch accents, modeled by operations on $\mathrm{Cf}$, and derived for each accent from their corresponding propositional effect as described in PH90.

The corollaries for pitch accented pronominals are: (1) when a pitch accent is applied to a pronominal, its main effect is attentional, on the order of items in Cf; (2) the obligation to accent a pronominal for attentional $r$ asons depends on the variance between what the text predicts and what the speaker would like to assert about the order of items in Cf.

These hypotheses arise from the following chain of assumptions:

(1) To analyze the effects of pitch accents on pronominals, it is necessary to distinguish between attentional and propositional salience. Attentional salience measures the degree to which an item is salient, expressible as a partial ordering, e.g., its ranking in Cf. It is a quantitative feature. In contrast, propositional salience, addressing an item's status in relation to mutual beliefs, is qualitative. It is calculated through inference chains that link semantic and pragmatic propositions.

Both attentional (Cf) and propositional (mutual beliefs) structures are updated throughout. However, unlike attentional structures which are ephemeral in various time scales and empty at the end of the discourse (Grosz and Sidner, 1986), mutual beliefs persist throughout the conversation, preserving at the end the semantic and pragmatic outcome of the discourse.

In addition, while propositions can be excluded from the mutual beliefs because they fail to meet some inclusion criterion, no lexical denotation is excluded from $\mathrm{Cf}$ regardless of its propositional value. This is because the salience most relevant to the attentional state is the proximity of a discourse entity to the head of Cf - the closer it is, the more it is centered and therefore, attentionally salient.

(2) Pitch accents on pronominals are primarily interpreted for what they say about attentional salience. One determiner of whether attentional or propositional effects are dominant is the type of information provided by the accented constituent. Because nonpronominals contribute discourse content, pitch accented nonpronominals are mainly interpreted with respect to the mutual beliefs, that is, for their propositional content. However, pronominals, with little intrinsic semantics, perform primarily an attentional function. Therefore pitch accented pronominals are mainly interpreted with respect to $\mathrm{Cf}$, for their attentional content.

(3) The specific attentional consequences of each 
pitch accent on pronominals can be extrapolated by analogy from the propositional interpretations in PH90, by replacing mutual beliefs with $C f$ as the salient set. Thus,

- $\mathrm{H}^{*}$ indicates instantiation of the pronominal's cospecifier as the $\mathrm{Cb}$, while $\mathrm{L}^{*}$ fails to instantiate it as the $\mathrm{Cb}$;

- The partially ordered set (salient scale) invoked by $\mathrm{L}+\mathrm{H}$ is $\mathrm{Cf}$;

- The inference path evoked by $H+L$ is, for attentional purposes, a traversal of $\mathrm{Cf}$.

(4) And therefore, the attentional effect of pitch accents can be formally expressed as an effect on the order of items in $C f$.

From these assumptions, I derive the following attentional consequences for pitch accented pronominals:

- Only one pitch accent, $\mathrm{L}+\mathrm{H}^{*}$, selects a $\mathrm{Cb}$ other than that predicted by centering theory and thereby reorders $\mathrm{Cf}$.

- $\mathrm{L}^{*}+\mathrm{H}$ appears to support an impending reordering but does not compel it.

- By analogy, the remaining pitch accents, seem to either weaken or strengthen the current center's $\mathrm{Cb}$ status, but do not force a reordering.

\section{Availability of cospecifiers}

The attentional interpretations are constrained by what has been mutually established in the prior discourse, or is situationally evident. Therefore, while contrastive stress may be mandated when grammatical features select the wrong cospecifier, the accenting is only felicitous when there is an alternate referent available.

For example, in

(2) John introduced Bill as a psycholinguist and then he $L+H *$ insulted him.

$\mathrm{L}+\mathrm{H}^{*}$ indicates that he no longer cospecifies with John. If the hearer is hasty, she might select Bill as the new $\mathrm{Cb}$. However, this is not borne out by the unaccented him, which continues to cospecify with Bill. Since he and him cannot select the same referent, he requires a cospecifier that is neither John nor Bill. Because, the utterance itself does not provide a any other alternatives, he $\mathrm{h}_{+H *}$ is only felicitous (and coherent) if an alternate cospecifier has been placed in Cf by prior discourse, or by the speaker's concurrent deictic gesture towards a discourteous male.

\section{Conclusion and Future Work}

By combining Pierrehumbert and Hirschberg's (1990) analysis of intonational meaning with Grosz, Joshi and Weinstein's (1989) theory of centering in discourse, the attentional affect of pitch accents becomes evident, and the paradox of pitch accented pronominals unravels. My goal here is to develop an analysis and a line of inquiry and to suggest that my derivative claims are plausible, and even extensible to an attentional analysis of pitch accents on nonpronominals. The proof, of course, will come from investigation by multiple means - constructed examples (e.g., Cahn, 1990), computer simulation, empirical analysis of speech data (e.g., Nakatani, 1993), and psycholinguistic experiments.

\section{References}

Dwight Bolinger. A Theory of Pitch Accent in English. Word, 14(2-3):109-149, 1958.

Susan E. Brennan, Marilyn W. Friedman, and Carl J. Pollard. A Centering Approach to Pronouns. Proceedings of the 25th Conference of the Association for Computational Linguistics, 1987.

Janet Cahn. The Effect of Intonation on Pronoun Referent Resolution. Draft, 1990. Available as: Learning and Common Sense TR 94-06, M.I.T. Media Laboratory.

Herbert H. Clark and Catherine R. Marshall. Definite Reference and Mutual Knowledge. In Webber, Joshi and Sag, editors, Elements of Discourse Understanding. Cambridge University Press, 1981.

Barbara Grosz, Aravind K. Joshi, and Scott Weinstein. Providing a unified account of definite noun phrases in discourse. Proceedings of the 21st Conference of the Association for Computational Linguistics, 1983.

Barbara J. Grosz, Aravind K. Joshi, and Scott Weinstein. Towards a Computational Theory of Discourse Interpretation. Draft, 1989.

Barbara J. Grosz and Candace L. Sidner. Attention, Intentions, and the Structure of Discourse. Computational Linguistics, 12(3):175-204, 1986.

George Lakoff. Presupposition and relative wellformedness. In Danny D. Steinberg and Leon A. Jakobovits, editors, Semantics: An Interdisciplinary Reader in Philosophy, Linguistics and Psychology, Cambridge University Press, 1971.

Christine Nakatani. Accenting on Pronouns and Proper Names in Spontaneous Narrative. Proceedings of the European Speech Communication Association Workshop on Prosody, 1993.

Janet B. Fierrehumbert. The Phonology and Phonetics of English Intonation. Ph.D. thesis, Massachusetts Institute of Technology, 1980.

Janet B. Pierrehumbert and Julia Hirschberg. The Meaning of Intonation Contours in the Interpretation of Discourse. In Philip R. Cohen, Jerry Morgan, and Martha E. Pollack, editors, Intentions in Communication, MIT Press, 1990.

Candace L. Sidner. Focusing in the Comprehension of Definite Anaphora. In Barbara J. Grosz, Karen Sparck-Jones, and Bonnie Lynn Webber, editors, Readings in Natural Language Processing, Morgan Kaufman Publishers, Inc., 1986. 